\title{
Cyclodextrin inclusion complexation and pharmaceutical applications
}

\author{
Helmut Viernstein ${ }^{\mathrm{a}}$, Peter Wolschann ${ }^{\mathrm{b}, *}$ \\ a Department of Pharmaceutical Technology and Biopharmaceutics, University of Vienna, \\ Vienna 1090 Austria \\ b Institute of Theoretical Chemistry, University of Vienna, Vienna 1090 Austria
}

*Corresponding author, e-mail: Karl.Peter.Wolschann@univie.ac.at

Received 15 Jun 2020

Accepted 20 Jun 2020

\begin{abstract}
Cyclodextrins are widely used in pharmacy, chemistry and other scientific disciplines, due to their unique properties which are consequences of the special geometries of these compounds. The cyclic arrangements of glucopyranose rings form structures where small and medium-sized molecules can be included. This inclusion reaction is of high interest, because it may change the physico-chemical properties of the guest molecules and allows the application of the involved compounds like drugs for a better delivery. Another important feature is the fact, that a large number of cyclodextrin derivatives is existing, with different affinities to the guest molecules, different thermodynamic properties and consequently a broad variety of applications. In the present review a short overview will be given about the various structures, the applications, in particular as drug carriers.
\end{abstract}

KEYWORDS: cyclodextrin, cyclodextrin derivatives, thermodynamics, solubility enhancement, drug carrier

\section{INTRODUCTION}

Cyclodextrins (cyclomaltooligosaccharides, CDs) are cyclic oligosaccharides consisting of glucopyranose subunits linked by $\alpha-1,4-$ glycosidic bonds. The so called "natural" CDs consist of six (cyclomaltohexaose, $\alpha-C D$ ), seven (cyclomaltoheptaose, $\beta$-CD) and eight (cyclomaltooctaose, $\gamma$-CD) can be obtained from enzymatic degradation of starch by cyclodextrin glucosyltransferase (CGTase). The arrangement of these linked glucopyranose units leads to the formation of a ring. The shape of these cyclic compounds depends on the number of glucose units (n). (n) Primary hydroxyl groups are located on one side of the rim at the cone, whereas $2 \mathrm{n}$ secondary hydroxyl groups are arranged on the opposite side of the ring. A more apolar cavity consisting of $\mathrm{CH}$ groups and glycosidic oxygen atoms is formed as a consequence of this particular structure with an average diameter of $5 \AA$ ( $\alpha$-CD), $6.2 \AA(\beta-C D)$ and $7.9 \AA(\gamma-C D)$, respectively and a thickness around $8 \AA$ [1-5].

Due to their unique structure CDs are able to include small or medium-sized organic molecules. This opens a huge field of applications in many scientific disciplines, in particular as recipients in pharmaceutical industry as drug carriers, for solubility enhancement and drug delivery. On the

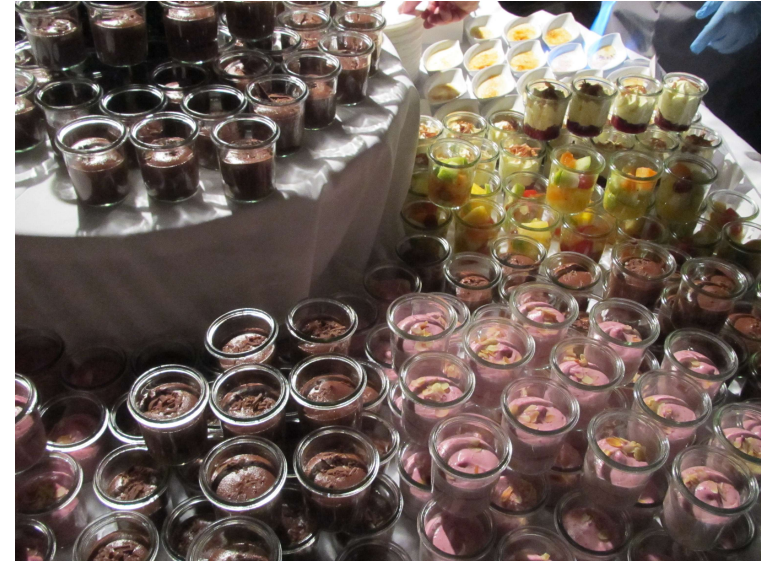

Fig. 1 Deserts with $\alpha$-cyclodextrin as additive (Cyclodextrin conference dinner, Saarbruecken 2014).

other hand the association protects the molecules included in the cavity, leading to a stabilization of the compounds against oxidation or interaction with light, or masking of taste and odour. Moreover, chemical properties are influenced, like the reactivity of the compounds, intramolecular processes and spectral features. Some CDs are also used in food (Fig. 1). 


\section{Structure of CDs}

The structures of the natural CDs were elucidated extensively by X-ray crystallography including cocrystallized water molecules $[6,7]$. Intra- and intermolecular hydrogen bonds can be found in the cavities and around the molecules, influencing the solubility of CDs. It has been observed that $\beta$-CD is less soluble in water than other CDs (nine to eleven times less soluble than other $\mathrm{CDs}$ ). In addition to a somewhat higher rigidity, intramolecular hydrogen bonding leads to a lower solubilization entropy for $\beta$-CD [8]. The influence of hydrogen bonding on the solubilities of CDs is strongly supported by the observation, that the solubility of all CDs is lower in $\mathrm{D}_{2} \mathrm{O}$, where the entropy-enthalpy balance, involved in the dissolution is less favorable. The solubility of CDs is also a consequence of self-assembling in solution [9]. All these findings are consequences of the strong interaction of CDs with water molecules, partly as solvating molecules but also bound to the interior of CDs.

Beyond the three "natural" CDs, a large number of chemically modified $\mathrm{CD}$ derivatives have been synthesized in order to extend the physico-chemical properties and the inclusion capacity of the parent CDs. Larger ring CDs have been synthesized and investigated in detail [10]. Methylated CDs and hydroxypropyl-CDs are widely synthesized as well as many different mono-substituted CDs.

The shape of the CD's interior differs depending on the number of glucopyranose units. However, substitution e.g. at the hydroxyl groups varies the inclusion features of CDs, not only through the size and the polarity of their cavities but also by the distinguishable flexibilities of the CDs themselves. For example, full methylation of the primary and secondary hydroxyl groups causes a marked distortion of the $\mathrm{CD}$ ring because it abrogates the intramolecular hydrogen bonds to some extent $[11,12]$. The resulting change of the shape of the cavity affects the geometries of the molecules. Most of the $\mathrm{CD}$ derivatives show a pronounced water solubility and moreover, interestingly for 2,6-dimethyl- $\beta$-CD a negative temperature coefficient of its solubility is observed [13].

Theoretical concepts have been applied to analyze the structural features of various CDs using empirical calculations methods. A wide range of symmetric and non-symmetric shape of structures were found $[14,15]$. Semiempirical methods have been also used for exploring the molecular geometries, but the correct description of hydrogen bond- ing depends on the methods used, which makes the obtained results doubtful to some extent. Increasing computational facilities make it possible to apply $\mathrm{ab}$ initio and density functional theory methods to describe structures and energy differences between various conformations of CDs in the gas phase. Symmetric structures with homodromic intramolecular hydrogen bonds at the rim have been found [16], the global minimum as a closed form with two hydrogen bond rings could not be evaluated experimentally up to now, probably because of experimental difficulties.

As the solvent plays an essential role for the geometries of CDs, empirical force field-based molecular dynamics methods are the most important methods for the determination of the geometries and the investigation of the dynamical behavior of CDs alone as well as for $\mathrm{CD}$ inclusion complexes. An extensive MD study on $\beta$-CD in water shows the presence of $3-5$ slightly bound water molecules inside the cavity $[17,18]$. A detailed analysis of the conformation and the conformational changesderived entropy is presented in the paper of Suarez and Diaz [19] not only for the parent CDs but also for a $\beta-C D$ inclusion complex. They performed a quite extended $5 \mathrm{~ms}$ MD simulation on the geometries to explore the dynamics and the conformational space of the natural CDs. A survey about MD simulations and also other theoretical molecular modeling methods, mainly of CD complexes is given [20].

Particularly, large ring CDs appeared to be a challenge for the MD simulations, as the flexibility of these ring systems is rather complicated. Such large ring systems were investigated by Gotsev et al [21]. Another systematic study was performed on CD10 (10 glucopyranose subunits in the ring system), where various force fields have been tested by replica exchange molecular dynamics [22]. Flipping of the glucopyranose subunits in the ring system in dependence on the temperature was observed and analysed. The same method was applied on CD14 ring system, where the various modes of intramolecular motion could be identified [23].

Beyond the three "natural" CDs, and the large ring systems, chemically modified $\mathrm{CD}$ derivatives have been developed in order to extend the physicochemical properties and the inclusion capacity of CDs. A survey of various CD derivatives is given elsewhere [24]. Evidently, the shape of the CD's interior differs depending on the number of glucose units, but, however, substitution, e.g. at the hydroxyl groups varies the inclusion properties of the 


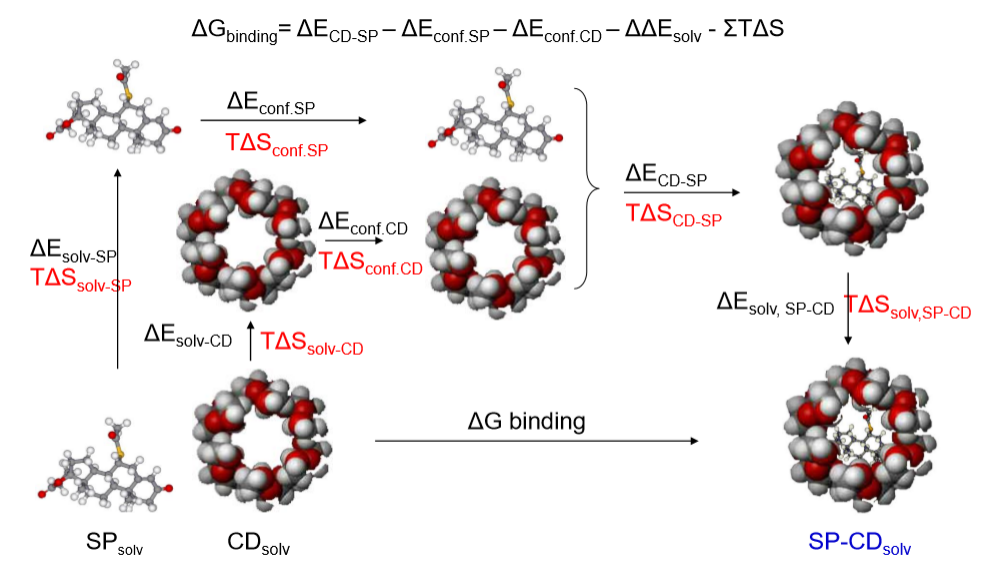

Fig. 2 Elementary steps of the inclusion reaction of spironolactone and $\beta$-cyclodextrin. The energies of each step are given. The total solvation energy $\left(\Delta \Delta \mathrm{E}_{\text {solv }}\right)$ consists of the three solvation processes. The sum of the entropic contributions $(\Sigma \mathrm{T} \Delta \mathrm{S})$ is the summation of all entropic terms.

ring systems, not only by the size and polarity of their cavities but also by different flexibilities and distortion of the systems. Hydroxypropyl substitution at one hydroxyl group of each glucose unit results in an interesting $\mathrm{CD}$ which is also used rather often [25]. The main problem of many substituted CDs is the fact, that the purification is rather difficult and so, particular on industrial scale, the available CDs are mixtures with an overall average value of substitution. However, the solubility of such compounds is high and in most cases the complexation affinity is larger than that of the parent compounds.

\section{Cyclodextrin inclusion complexation}

A wide variety of small and medium-sized molecules can be included in the CDs cavities. No covalent bond is formed, the association or "host-guest inclusion complexation" is a consequence of the interaction of the guest molecules with the cavity of the host molecules. The specificity of this "molecular recognition" lies between unspecific interactions of a solute with the solvent - solvation - and highly specific receptor interactions $[26,27]$. The driving forces for the inclusion complexation are hydrophobic, van der Waals and electrostatic interactions as well as hydrogen bond formation abilities [28].

The association complexation changes the properties of the guest molecules to a large extent which is broadly used in many applications of CDs in environmental and technical chemistry, in biology and food chemistry, as well as in pharmacy and pharmaceutical technology. Stereospecific separations of diastereomers and optical isomers, extraction of natural products, protection of unstable compounds, e.g. light-, temperature- or oxidation-sensitive substances, masking of odour or taste are caused by complexation with CDs. Moreover, emulsification of highly polar compounds, modifications of catalytic activities and support in organic syntheses are topics which make CDs indispensable excipients in many scientific disciplines.

The association constants and the subsequent thermodynamic parameters characterize the binding ability of included compounds. The overall binding constants can be measured by many different methods. One most popular is the solubility method where the equilibrium constant can be calculated from concentration of the guest molecule with rising concentration of $\mathrm{CD}$ [29]. Another rather efficient method is the use of caloric methods, e.g. isothermal calorimetry $[30,31]$. The overall inclusion reaction consists of several elementary steps. A scheme of these elementary steps together with the corresponding thermodynamic is given in Fig. 2 for the complexation of spironolactone, a compound of rather low solubility and of very high affinity to $\beta$ $\mathrm{CD}$. After dissolution of solid spironolactone, the solvation shell has to be removed and conformational change need to take place before the compound is able to interact with the CD's interior [32]. The energetic parameters of these elementary steps are given by $\Delta \mathrm{H}_{\text {solv }}, \Delta \mathrm{E}_{\text {solv-SP }}$ and $\Delta \mathrm{E}_{\text {conf,SP }}$ in Fig. 2 .

For $\mathrm{CD}$ the solvation shell and the water molecules inside the cavity have to be removed, too $\left(\Delta \mathrm{E}_{\text {solv }}\right)$, followed by the final solvation of the complex $\left(\Delta \mathrm{E}_{\text {solv }}, \mathrm{SP}-\mathrm{CD}\right)$. Each elementary step is associated with an energy difference accompanied 
by reaction entropy changes. Most of the single processes depend on the guest molecular and the $\mathrm{CD}$ used. Under special circumstances changes of the energetics of the individual steps result in changes of the reaction mechanisms. Thus, the inclusion reaction of spironolactone exhibits a rather high complexation constant. Enthalpy-entropy compensation is observed for $\beta$-CD, whereas for 2,6-dimethyl- $\beta$ $\mathrm{CD}$ the contribution of the reaction entropy to the overall free energy is of opposite sign [32].

Numerous papers have been published describing the thermodynamics of the interaction between CDs and guest molecules [33,34]. In particular, many thermodynamic data of organic compounds complexed to CDs are given in investigations published by Rekharsky $[35,36]$. The experimentally observed complexation constants range over six order of magnitude. The overall reaction enthalpies and entropies can be determined from the temperature dependence of the equilibrium constants or from calorimetric experiments for the detailed characterization of the complexes. The reaction entropy plays an essential role in the thermodynamics of this type of bimolecular reactions. Enthalpy-entropy compensation is recognized in many cases [37].

Based on the large amount of available experimental data, models for predicting the association constants or the related free energies of complexation have been developed. The aim of such studies is not only to select convenient CDs for a potent complexation of a distinct compound, but also to get some insight into the parameters influencing the affinity between host and guest molecules. Prediction models for the free energy of complexation using several molecular descriptors were established $[38,39]$, an approach predicting the binding capacity was published just recently [40], and also artificial neural networks were used to predict affinity of hosts-guest interactions [41]. Most of these investigations consider only basic CDs.

As already mentioned before the overall reaction of the bimolecular process consists of many elementary steps. Depart from the scheme given in Fig. 2 the reaction between a compound and CDs follows many local minima on the energy hypersurface, before the global minimum, the energetically stable inclusion complex, is reached. A careful analysis of the reaction path of the inclusion of $\alpha$-mangostin into $\beta$ cyclodextrin, 2,6-dimethyl- $\beta$-cyclodextrin (DM $\beta \mathrm{CD}$ ) and hydroxypropyl- $\beta$-cyclodextrin (HP $\beta C D$ ) was performed just recently [42]. By an extensive Molecular Dynamics simulation (over 500 ns sim- ulation time in water) it was shown, that on the reaction pathway from the isolated molecule many association complexes were formed, where the guest compound is located at various positions outside the CDs rim as well as at different geometries inside the cavity. Moreover, it could be recognized, that the reaction diagram was different for the individual $\mathrm{CDs}$, also because the binding free energies are remarkably different for the three CDs. DM $\beta C D$ forms stronger inclusion complexes as HP $\beta C D$. $\beta$-CD forms the weakest complexes of the three considered CDs. This finding was proven also experimentally, where the measured association constants follow the same trend.

\section{Drug-cyclodextrin complexes}

The importance of the applications of CDs in pharmacy is discussed in many reviews. A review on historical perspectives of the use of CDs in pharmacy is published [43]. General overviews are given in a series of review articles [44-48]. Other extended reviews describe the solubility enhancement of poorly water-soluble drugs and the subsequent increase of bioavailability and efficiency of the active compounds as well as facilitating of their controlled release in numerous investigations [49-52]. Special applications of CD inclusion complexation are also described for many cases [53-55]. In the pharmaceutical fields, CDs were widely used to improve the stability and solubility of the insoluble drugs in water or organic solvent-water mixtures through molecular encapsulation. The ability of encapsulation of CDs with drugs strongly depends on the structural nature of CDs.

The structures of drug-CD inclusion complexes are investigated and described in a huge number of publications, some recent crystallographic studies as well as Molecular Dynamics investigations are given here [56-59]. As the inclusion complexation of CDs is used to improve the solubility of apolar compounds with low solubility in water the concentration of inclusion complexes is in many cases much higher than the saturation concentration of the compounds alone. The dissolution rate of the considered compounds depends on many parameters, like particle size, stirring of the heterogenous solution, temperature and so on.

The mechanism of CD inclusion of low soluble compounds is rather complicated. As an example the pronounced solubility enhancement of nabilone should be mentioned. Nabilone is a synthetic cannabinoid and is used against nausea caused by chemotherapy treatment $[60,61]$. The solubility in 


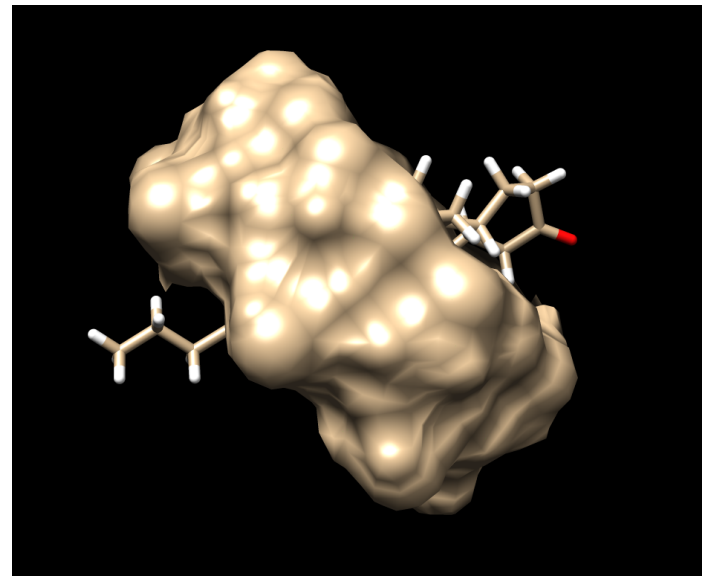

Fig. 3 Inclusion complex of nabilone and 2,6-dimethyl- $\beta$ cyclodextrin. The minimized (M06-2X/6-31G(d,p) structure of the complex is given. Nabilone in sticks, the solid surface of the CD derivate (USCF Chimera 1.14)

pure water is lower than $10^{-6} \mathrm{~mol} / 1$. The solubility is increased to some extent by encapsulation by $\beta$ $\mathrm{CD}$, but complexation with 2,6-dimethyl-CD leads to a tremendous higher concentration of the drug [62]. A structure of the complex between nabilone and 2,6-dimethyl-CD obtained from quantum mechanical minimization (M02-2X/6-31G(p,d)) is depicted in Fig. 3.

The dissolution of a low soluble compound is performed by stirring the heterogenous system (solid compound and CD solution) for several hours. In the case of nabilone first an increase of the concentration of nabilone is observed but later on the concentration of the complex - and of nabilone - is decreased again. The reason for this behaviour results from the formation of several different aggregates between CDs as well as between CD complexes $[9,47]$, some have a rather low solubility which leads to precipitation of the complexes aggregates. A scheme of possible aggregates is given in Fig. 4.

Such a solubilization behaviour is also observed for other cannabinoids [63]. Another example is the solubility profile of dexamethasone where the $\gamma$-CD inclusion complex has only a limited solubility and therefore, this complexation does not lead to an increase of the concentration of the drug. The application of randomly substituted hydroxypropyl- $\gamma-\mathrm{CD}$ in contrary leads to a tremendous increase of the drug concentration in s olution $[47,64,65]$. Interesting cyclodextrin derivatives are also cyclodextrin derivatives with different amounts of substitutions of sulfobutylether sodium salt. These compounds are applied to increase the solubility of many drug molecules $[66,67]$.

A broad field of applications of CD inclusion complexation is opened by the copolymerization of $\beta$-CD. The polymerization with many different monomers, with different functional groups and cross-linkers leads to a series of compounds with various properties. A review about these compounds and their applications has been published recently [68].

\section{Cyclodextrin-drug complexes at the cellular membrane}

Solubility enhancement and subsequent increase of bioavailability are the main aims of the use of CDs as pharmaceutical recipients. Beyond this increase of the concentration of biologically active compounds, CDs may also support the transfer of drugs through the cellular membranes. CDs and CD complexes are not able to penetrate the membrane system without destroying the membrane structure, but they interact with the membrane surface [69-71]. They influence e.g. the composition of the membrane by extraction of cholesterol. The transport of drugs to the lipid bilayer surface has been investigated in many studies. In a recent study is has been shown, that various cyclodextrin derivatives interact in a different way at the membrane surface $[72,73]$, and enable the penetration of the drug through the membrane to some extent.

\section{Cyclodextrins as medicinal applications}

For a number of years it is already known that cyclodextrins (CDs) can be used not only as excipients in drugs but also as active pharmaceutical ingredients (API) for special diseases. The most important mechanism of action is the complex binding of CDs with cholesterol located in biomembranes followed by the complexation of drug substances. For native $\mathrm{CDs}$ as well as some derivatives, like hydroxypropylated $\mathrm{CD}$, randomly methylated $\mathrm{CD}$ or modified gamma-CD (Sugammadex) biological activities are proven.

For oral administration of native CDs there are almost no restrictions, according to the rules of the Food and Drug Administration (FDA) they have "GRAS" status which means that they are recognized as save for human use; only the intake of $\beta$-CD is limited to a maximum of $5 \mathrm{mg} / \mathrm{kg} /$ day. $\alpha-C D$ was included in the European Union Register on nutrition and health claims in 2013 and approved for reduced post-meal glycemic responses [74]. For 


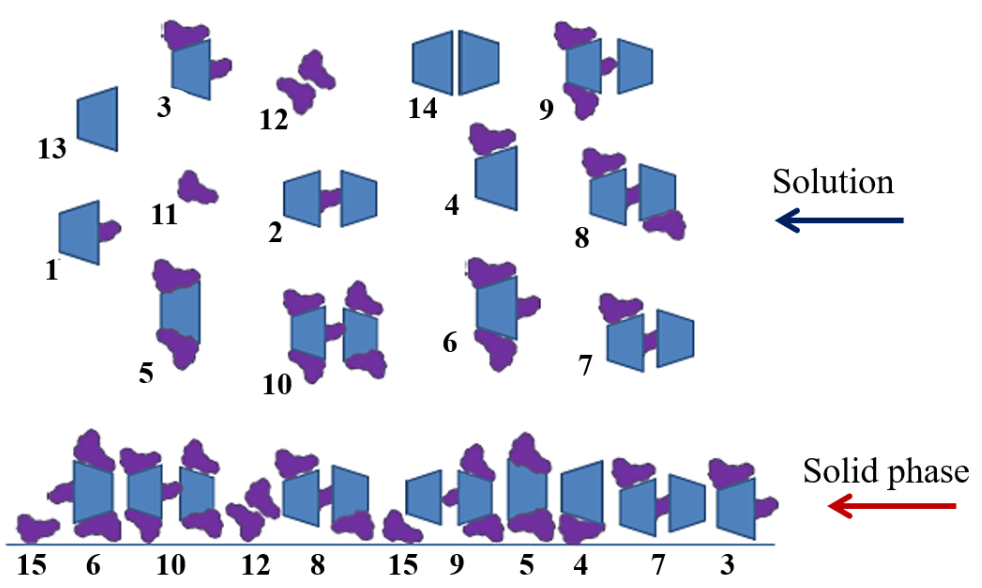

Fig. 4 Scheme of the various aggregations of nabilone - 2,6-dimethyl- $\beta$-CD. 1 and 2 : inclusion complex (1:1, 2:1), 3 to 10 association complexes, 11 nabilone in solution, 12 nabilone dimer, $13 \mathrm{CD}, 14 \mathrm{CD}$ dimer, 15 solid nabilone. Some of these aggregates might have a lower solubility. Colored in blue $=\mathrm{CD}$, colored in purple $=$ nabilone molecule.

parenteral applications native CDs are not recommended by the European Medicines Agency (EMA), only 2 -hydroxypropyl- $\beta$-CD, 2-hydroxy-propyl- $\gamma$-CD or Sugammadex are approved as biocompatible excipients or drug substances.

Hereafter, only examples of successful medical applications in humans are described in details.

Due to the high binding capacity of native CDs and modified CDs to cholesterol they are good candidates for antiviral drug substances; such a new type of an antiviral drug may disrupt the outer shell of the virus and destroys the integrity of the particle [75]. CDs modified with mercaptoundecane sulfonic acid are highly biocompatible and showed in vitro and in an animal model a limitation of viral replication, including herpes simplex virus, respiratory syncytial virus, Dengue virus and Zika virus $[76,77]$. All these promising data could be a first step in the development of a vaccine against the new coronavirus.

Clinical trials are still ongoing with hydroxypropyl- $\beta$-cyclodextrin for the treatment of the rare kidney disease named Focal Segmental Glomerulosclerosis, where the $\mathrm{CD}$ removes excess cholesterol from the kidneys [78].

The Niemann-Pick Type 1 disease (NPC1) is a progressive autosomal recessive disease caused by defective intracellular cholesterol and lipid trafficking. Currently, there are no therapies approved by FDA or EMA, but it could be shown that hydroxypropyl- $\beta$-cyclodextrin given by intrathecal injections results in a stabilization of the neurological functions. Though the mechanism of action remains unknown, there is evidence that the cy- clodextrin binds to lysosomal cholesterol [79]. Orphan designation for hydroxypropyl- $\beta$-cyclodextrin had been granted in the United States and in the European Union [80].

The modified $\gamma$-cyclodextrin Sugammadex (Bridion $\AA$; Cyclooctakis- $(1 \rightarrow 4)$-[6-S-(2-carboxyethyl)-6- $\alpha$-D-glucopyranosyl] sodium salt) is used to reverse the neuromuscular blockade of the muscle relaxants rocuronium bromide and vecuronium bromide in adults undergoing surgery. It is given as a single bolus injection intravenously up to $4 \mathrm{mg} / \mathrm{kg}$ body weight. The date of issue of marketing authorization valid throughout the European Union was 07/2008 [81].

\section{REFERENCES}

1. Szejtli J (1998) Introduction and general overview of cyclodextrin chemistry. Chem Rev 98, 1743-1754.

2. Szejtli J (2004) Past, present and future cyclodextrin research. Pure Appl Chem 76, 1825-1845.

3. Szejtli J, Osa T (1996) Comprehensive Supramolecular Chemistry Vol 3, Cyclodextrins, Pergamon Press, Oxford.

4. Dodziuk H (2006) Cyclodextrins and Their Complexes, Wiley-VCH Verlag GmbH, Weinheim.

5. Crini G (2014) Review: A history of cyclodextrins. Chem Rev 114, 10940-10975.

6. Saenger W, Jakob J, Gessler K, Steiner T, Hoffmann D, Sanabe H, Koizumi K, Smith SM, et al (1998) Structures of the common cyclodextrins and their larger analogues - beyond the doughnut. Chem Rev 98, 1787-1802.

7. Saenger W, Steiner T (1998) Cyclodextrin inclusion complexes: Host-guest interactions and hydrogenbonding networks. Acta Cryst A 54, 798-805. 
8. Jin S, Cui X, Qi Y, Shen Y, Li H (2019) Measurement and correlation of the solubility of $\beta$-cyclodextrin in different solutions at different temeperatures and thermodynamic study of the dissolution process. Processes 7, ID 135.

9. Loftsson T, Saokham P, Sa Couto AR (2019) Selfassociation of cyclodextrins and cyclodextrin complexes in aqueous solutions. Int $J$ Pharm 560, 228-234.

10. Taira H, Nagase H, Endo T, Ueda H (2006) Isolation, purification and characterization of large-ring cyclodextrins (CD36 similar to CD39) J Incl Phenom Macrocycl Chem 56, 23-28.

11. Jacob J, Gessler K, Hoffmann D, Sanbe H, Koizumi K, Smith SM, Takaha T, Saenger W (1999) Band-flip and kink as novel structural motifs in alpha- $(1 \rightarrow 4)$ D-glucose oligosaccharides. Crystal structures of cyclodeca- and cyclotetradecaamylose. Carbohydr Res 322, 228-246.

12. Aree T, Saenger W, Leibnitz P, Hoier H (1999) Crystal structure of heptakis(2,6-di-O-methyl)-betacyclodextrin dihydrate: a water molecule in an apolar cavity. Carbohydr Res 315, 199-205.

13. Kusmin A, Lehner RE, Kammel M, Saenger W (2008) Native and methylated cyclodextrins with positive and negative solubility coefficients in water studied by SAXS and SANS. $J$ Phys Chem $B$ 112, 12888-12898.

14. Lipkowitz KB (1998) Applications of computational chemistry to the study of cyclodextrins. Chem Rev $\mathbf{9 8}$ 1829-1874.

15. Kozar V, Venanzi CA (1997) Reconsidering the conformational flexibility of $\beta$-cyclodextrin. $J$ Mol Struct (Theochem) 395, 451-468.

16. Snor W, Liedl E, Weiss-Greiler P, Karpfen A, Viernstein $\mathrm{H}$, Wolschann P (2007) On the structure of anhydrous $\beta$-cyclodextrin. Chem Phys Lett 441, 159-162.

17. Lawtrakul L, Viernstein H, Wolschann P (2003) Molecular dynamics simulations of $\beta$-cyclodextrin in aqueous solution. Int $J$ Pharm 256, 33-41.

18. Boonyarattanakalin KS, Wolschann P, Lawtrakul L (2011) Molecular dynamics of $\beta$-CD in water/cosolvent mixtures. $J$ Incl Phenom Macrocycl Chem 70, 279-290.

19. Suarez D, Diaz N (2017) Conformational and entropy analyses of extended molecular dynamics simulations of $\alpha$-, $\beta$ - and $\gamma$-cyclodextrins and of the $\beta$ cyclodextrin/nabumetone complex. Phys Chem Chem Phys 19, 1431-1440.

20. Quevedo MA, Zoppi A (2018) Current trends in molecular modeling methods applied to the study of cyclodextrin complexes. J Incl Phenom Macrocyl Chem 90, 1-14.

21. Gotsev MG, Ivanov PM, Jaime C (2007) Molecular dynamics study of the conformational dynamics and energetics of some large-ring cyclodextrins (CDn, $\mathrm{n}=24,25,26,27,28,29)$. Chirality 19, 203-213.
22. Khuntawee W, Rungrotmongkol T, Wolschann P, Pongsawasdi P, Kungwan H, Okumura S, Hannongbua $S$ (2016) Conformation study of $\varepsilon$-cyclodextrin: Replica exchange molecular dynamics. Carbohydr Polym 141, 99-105.

23. Khuntawee W, Kunaseth $M$, Rungmin Ch, Intagorn S, Wolschann P, Kungwan N, Rungrotmongkol T, Hannongbua S (2017) Comparison of implicit and explicit solvation models for iota-cyclodextrin conformation analysis from replica exchange molecular dynamics. $J$ Chem Inf Mod 57, 778-786.

24. Szente L, Szejtli (1999) Highly soluble cyclodextrin derivatives: chemistry, properties, and trends in development. Adv Drug Deliv Rev 36, 17-28.

25. Kerdpol K, Kincuntod J, Wolschann P, Mori S, Rungnim C, Kunaseth M, Okumura H, Kungwan N, et al (2019) Cavity closure of 2-hydroxypropyl- $\beta$ cyclodextrin: Replica exchange molecular dynamics simulations. Polymers 11, ID 145.

26. Caira MR (2019) Cyclodextrin inclusion of medicinal compounds fo enhancement of their physicochemical and biopharmaceutical properties. Curr Top Med Chem 19, 2357-2370.

27. Crini G, Fourmentin S, Fenyvesi E, Torri G, Fourmentin M, Morin-Crini N (2018) Cyclodextrins, from molecules to applications. Environ Chem Lett 16, 1361-1375.

28. Liu L, Guo QX (2002) Th driving force in the inclusion complexation of cyclodextrins. $J$ Incl Phenom Macrocycl Chem 42, 1-14.

29. Higuchi T, Connors KA (1965) Phase-solubility techniques. Adv Anal Chem Instr 7, 117-212.

30. Kfoury M, Landy D, Fourmentin S (2018) Characterization of cyclodextrin/volatile inclusion complexes: A review. Molecules 23, ID 1204.

31. Kairys V, Baranauskiene L, Kazlaukiene M, Matulis D, Kazlauskas E (2019) Binding affinity in drug design: experimental and computational techniques. Expert Opin Drug Discov 14, 755-768.

32. Weinzinger P, Weiss-Greiler P, Snor W, Viernstein $\mathrm{H}$, Wolschann P (2007) Molecular dynamics simulations and quantum chemical calculations on $\beta$ cyclodextrin - spironolactone complex. J Incl Phenom Macrocycl Chem 57, 29-33.

33. Köhler G, Viernstein H, Wolschann P (1996) Molecular calculations and thermodynamical considerations on the solubility enhancement of triflumizole by cyclodextrin complexation. J Incl Phenom Macrocycl Chem 25, 237-241.

34. Wankar J, Kotla NG, Gera S, Rasala S, Pandit A, Rochev YA (2020) Recent advances in host-delivery and biomedical engineering. Adv Funct Materials, ID 1909049.

35. Rekharsky MV, Inoue Y (1998) Complexation thermodynamics of cyclodextrins. Chem Rev 98, 1875-1918.

36. Rekharsky MV, Inoue Y (2000) Chiral recognition 
thermodynamics of $\beta$-cyclodextrin: the thermodynamic orign of of enantioselectivity and the enthalpyentropy compensation effect. J Amer Chem Soc 122, 4418-4435.

37. Schonbeck C, Hol R (2019) Exploring the origins of enthalpy-entropy compsensation by calorimetric studies of cyclodextrin complexes. $J$ Phys Chem $B$ 123, 6686-6693.

38. Klein CT, Polheim D, Vierntein $\mathrm{H}$, Wolschann $\mathrm{P}$ (2000) A method for predicting the free energies of complexation between $\beta$-cyclodextrin and guest molecules. J Incl Phenom Macrocycl Chem 36, 409-423.

39. Klein CT, Polheim D, Vierntein $H$, Wolschann $P$ (2000) Predicting the free energies of complexation betwen cyclodextrins and guest molecules: Linear versus nonlinear models. Pharm Res 17, 358-365.

40. Di P, Chen JH, Liu LP, Li WH, Tang Y, Liu GX (2020) In silico prediction of binding capacity and interaction forces of organic compounds with alpha- and betacyclodextrins. J Mol Liquids 302, ID 112585.

41. Xu Q, Wei C, Liu RN, Gu SJ, Xu J (2015) Quantitative structure-property relationship study of betacyclodextrin complexation free energies of organic compounds. Chemometr Intel Lab Systems 146, 313-321.

42. Hotarat W, Rungnim C, Wolschann P, Kungwan N, Rungrotmongkol T, Hannongbua S (2019) Encapsulation of alpha-mangostin and hydrophilic betacyclodextrins revealed by all-atom molecular dynamics simulations. J Mol Liqu 288, ID 110965.

43. Uekama K, Hirayama F, Irie T (1998) Cyclodextrin carrier systems. Chem Rev 98, 2045-2076.

44. Challa R, Ahuja A, Ali J, Khar RK (2005) Cyclodextrins in drug delivery: An updated review. AAPS Pharm Sci Tech 6, E329-E357.

45. Loftsson T, Duchene D (2007) Cyclodextrins and their pharmaceutical application. Int J Pharm 329, $1-11$.

46. Loftsson T, Jarho P, Masson M, Jaervinen T (2005) Cyclodextrins in drug delivery. Exp Opin Drug Deliv 2, 335-351.

47. Jansook P, Ogawa N, Loftsson T (2018) Cyclodextrins: structure, physicochemical properties and pharmaceutical applications. Int $J$ Pharm 535, 272-284.

48. Vikas Y, Sandeep K, Braham D, Majusha C, Budhwar V (2018) Cyclodextrin complexes: An approach to improve the physicochemical properties of drugs and applications of cyclodextrin complexes. Asian $J$ Pharm 12, S394-S409.

49. Oda M, Saitoh H, Kobayashi M, Aungst BJ (2004) $\beta$ cyclodextrin as a suitable solubilizing agent for in situ absorption study of poorly water-soluble drugs. Int $J$ Pharm 280, 95-102.

50. Zhao J, Yang J, Xie Y (2019) Improvement strategies for the oral bioavailability of poorly water- soluble flavonoids: An overview. Int $J$ Pharm 570, ID 118642.

51. Carrier R, Miller L, Ahmed I (2007) The utility of cyclodextrins for enhancing oral bioavailability. $J$ Control Release 123, 78-99.

52. Brewster M, Loftsson T (2007) Cyclodextrins as pharmaceutical solubilizers. Adv Drug Deliv Rev 59, 645-666.

53. Tian B, Hua S, Liu J (2020) Cyclodextrin-based delivery systems for chemothera-peutic anticancer drugs: A review. Carbohydr Polym 232, ID 115805.

54. Conceicao J, Adeoye O, Cabral-Marques HM, Sousa Lobo JM (2018) Cyclodextrins as excipients in tablet formulations. Drug Discov Today 23, 1274-1284.

55. Abdolmaleki A, Ghasemi F, Ghasemi JB (2017) Computer-aided drug design to explore cyclodextrin therapeutics and biomedical applications. Chem Biol Drug Des 89, 257-268.

56. Aree $\mathrm{T}$ (2020) Beta-cyclodextrin encapsulation of nortriptyline $\mathrm{HCl}$ and amitriptyline $\mathrm{HCl}$ : Molecular insights from single-crystal X-ray diffraction and DFT calculations. Int J Pharm 575, ID 118899.

57. Christoforides E, Fourtaka K, Andreou A, Bethanis K (2020) X-ray crystallography and molecular dynamics studies of the inclusion complexes of geraniol in $\beta$-cyclodextrin, heptakis(2,6-di-O-methyl)$\beta$-cyclodextrin and heptakis(2,3,6-tri-O-methyl)- $\beta$ cyclodextrin. J Mol Struct 1202, ID 127350.

58. Kicuntod J, Khuntawee W, Wolschann P, Pongsawasdi P, Chavasiri W, Kungwan N, Rungrotmongkol T (2016) Inclusion complexation of pinostrobin with various cyclodextrin derivatives. $J \mathrm{Mol}$ Graph $\mathrm{Mod}$ 63, 91-98.

59. Papaioannou A, Christoforides E, Bethanis K (2020) Inclusion complexes of naringenin in dimethylated and permethylated $\beta$-cyclodextrin: Crystal structures and molecular dynamics studies. Crystals 10, ID 10.

60. Chow R, Valdez C, Chow N, Zhang DN, Im J, Sodhi E, Lock M (2020) Oral cannabinoid for the chemotherapy in induced nausea and vomitting - A systematic review and meta-analysis. Supp Care Cancer 28, 2095-2103.

61. Ware MA, Daeninck P, Maida V (2008) A review of nabilone in the treatment of chemotherapy-induced nausea and vomiting. Therapeut Clin Risk Managm 4, 99-107.

62. Viernstein $H$, Rungrotmongkol $T$, Wolschann $P$ (2020) Solubility enhancement of nabilone by inclusion complexation with 2,6-dimethyl- $\beta$-cyclodextrin. (in preparation).

63. Hazekamp A, Verpoorte R (2006) Structure elucidation of the tetrahydrocannabiol complex with randomly methylated b-cyclodextrin. Europ J Pharm Sci 29, 340-347.

64. Jansook P, Loftsson T (2008) $\gamma$-CD/HP $\gamma$-CD: Synergistic solubilization. Int $J$ Pharm 363, 217-219.

65. Loftsson T, Saokham P, Sa Couto AR (2019) Self- 
association of cyclodextrins and cyclodextrin complexes in aqueous solutions. Int $J$ Pharm 560, 228-234.

66. Brewster ME, Vandecruys R, Peeters J, Neeskens, Vereck G, Loftsson $T$ (2008) Comparative interaction of 2-hydroxypropyl- $\beta$-cyclodextrin and sulfobutylether- $\beta$-cyclodextrin with itraconazole: Phase-solubility behaviour and stabilization of supersaturated drug solutions. Eur J Pharm Sci 34, 94-103.

67. Das SK, Kahali N, Bose A, Khanam J (2018) Physicochemical characterization and in vitro dissolution performance of ibuprofen-CAPTISOL (sulfobutylether sodium salt of $\beta$-CD) inclusion complexes. J Mol Liqu 261, 239-249.

68. Tarannum N, Suhani, Kumar D (2020) Syntheses, characterization and applications of copolymer of $\beta$ cyclodextrin. $J$ Polymer Res 27, ID 89.

69. Hammoud Z, Khreich N, Auezova L, Fourmentin S, Elaissari A, Greige-Gerges H (2019) Cyclodextrinmambrane interaction in drug delivery and membrane structure maintenance. Int $J$ Pharm 564, 59-76.

70. Loftsson T, Brewster ME (2012) Cyclodextrins as functional excipients: Methods to enhance complexation efficiency. J Pharm Sci 101, 3019-3032.

71. Leclercq L (2016) Interactions between cyclodextrins and cellular components: Towards greener medical applications. Beilstein J Org Chem 12, 2644-2662.

72. El Achkar T, Moufawad T, Ruellan S, Landy D, GreigeGerges H, Fourmetin S (2020) Cyclodextrins: from solute to solvent. Chem Comm 56, 3385-3388.

73. Khuntawee W, Wolschann P, Rungrotmongkol T, Wong-Ekkabut J, Hannongbua S (2015) Molecular dynamics simulations of the interaction of beta cyclodextrin with lipid bilayer. $J$ Chem Inf Model 55, 1894-1902.

74. Hotarat W, Nutho B, Wolschann P, Rungrotmongkol T, Hannongbua S (2020) Delivery of alpha-mangostin using cyclodextrins through a biological membrane: Molecular dynamics simulation. Molecules 25, ID 2532.

75. EU Register on Nutrition and Health Claims, Commission Regulation (EU) 536/2013.

76. Braga SS (2019) Cyclodextrins: Emerging medicines of the new millennium. Biomolecules 9, ID 801.

77. Jones ST, Cagno V, Janecek M, Ortiz D, Gasilova N, Piret J, Gasbarri M, Constant DA, et al (2020) Modified cyclodextrins as broad-spectrum antivirals. Sci $A d v$ 6, ID 9318.

78. Mitrofanova A, Molina J, Santos JV, Guzman J, Morales XA, Ducasa GM, Bryn J, Sloan A, et al (2018) Hydroxypropyl-beta-cyclodextrin protects from kidney disease in experimental Alport syndrome and focal segmental glomerulosclerosis. Kidney Int 94, 1151-1159.

79. Feltes M, Gale SE, Moores S, Ory DS, Schaffer JE (2020) Monitoring the itinerary of lysosomal cholesterol in Niemann-Pick type C1-deficient cells after cyclodextrin treatment. J Lipid Res 61, 403-412.

80. European Medicines Agency (2015) Public summary of the opinion on orphan designation, Hydroxy-propyl-beta-cyclodextrin for the treatment of Niemann-Pick disease, type C, EMA/COMP/546608/2011 Rev. 1.

81. European Medicines Agency, Bridion, Sugammadex, available at: www.ema.europa.eu/en/medicines/ human/EPAR/bridion. 